\title{
EXPERIENCIA DE USUARIO EN TELEVISIÓN CONECTADA: UN ESTUDIO CON USUARIOS
}

\section{Verónica Mansilla y Mari-Carmen Marcos}

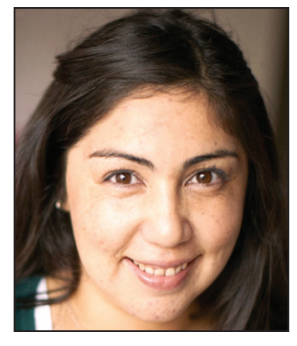

Verónica Mansilla es licenciada en comunicación social por la Universidad Andrés Bello en Chile (2005). Titulada en el máster en gestión de contenidos digitales por la Universitat de Barcelona y la Universitat Pompeu Fabra (2012).

http://orcid.org/0000-0002-8576-045X

Universitat de Barcelona Melcior de Palau, 140. 08014 Barcelona, España v.mansilla@gmail.com

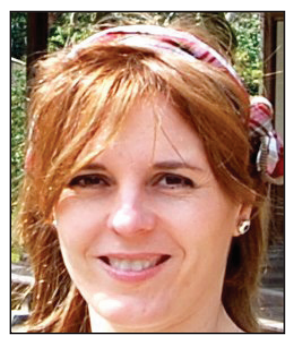

Mari-Carmen Marcos es profesora de documentación en el Depto. de Comunicación de la Univ. Pompeu Fabra. Su línea de investigación es la interacción persona-ordenador en sistemas de recuperación de información. Se ha especializado en el análisis de interfaces a través del estudio del comportamiento de lo usuarios. En los últimos años ha incorporado a sus trabajos la tecnología de seguimiento de la mirada (eye tracking).

http://orcid.org/0000-0002-9576-3830

Universitat Pompeu Fabra Roc Boronat, 138. 08018 Barcelona, España http://www.mcmarcos.com mcarmen.marcos@upf.edu

\section{Resumen}

Se presenta la metodología y resultados de un estudio con usuarios realizado sobre varios dispositivos de televisión conectada. Entre los métodos aplicados se encuentran la evaluación heurística, los tests de usuarios y la técnica de seguimiento de la mirada (eye tracking). Los resultados apuntan a una baja usabilidad en las tareas que requieren el uso del teclado, así como en aquellas relacionadas con el servicio de TV a la carta (video on demand, VoD). Estas tareas se han analizado en profundidad en el dispositivo que incorpora el protocolo HbbTV, para lo que se ha utilizado un eye tracker.

\section{Palabras clave}

Usabilidad, Experiencia de usuario, Televisión conectada, Pruebas con usuarios, Eye tracking, Evaluación heurística.

\section{Title: User experience in connected TV: a user study}

\section{Abstract}

Methodology and results of a user study on several connected TV devices is presented. Heuristic evaluation, user testing and eye tracker are applied. The results point out a low usability in the tasks that require keyboard and those related to the video on demand service (VoD). These latter tasks have been analysed in depth with eye tracker technology in the device that follows the HbbTV protocol.

\section{Keywords}

Usability, User experience, Connected TV, User testing, Eye tracking, Heuristic evaluation.

Mansilla, Verónica; Marcos, Mari-Carmen (2013). "Experiencia de usuario en televisión conectada: un estudio con usuarios". El profesional de la información, marzo-abril, v. 22, n. 2, pp. 122-127.

http://dx.doi.org/10.3145/epi.2013.mar.04

\section{Introducción}

El concepto de "televisión conectada" es relativamente reciente. Bill Gates fue uno de los primeros en referirse a ella en una sesión sobre web 2.0 que tuvo lugar en el Foro Económico Mundial de 2007 al afirmar que internet transfor- maría la televisión en los siguientes cinco años a causa de la unión entre ordenadores y televisores (El mundo, 2007).

Así, la televisión conectada es una combinación de televisión en el sentido tradicional de radiodifusión (broadcast) y banda ancha (broadband). Gracias a esta unión, desde el 
propio televisor se puede profundizar acerca de lo que se está viendo realizando una búsqueda en la Web, compartirlo en redes sociales, acceder a aplicaciones de muchos tipos, por ejemplo las de videoconferencia, pero también a los servicios de TV a la carta, es decir, a la programación ya emitida en las cadenas.

Su introducción en el mercado es reciente, apenas unos años, y cada marca trabaja en su producto sin que haya una estandarización y mucho menos un acuerdo, o al menos unos principios o pautas, en cuanto a sus interfaces. Así, podemos encontrar modelos de Sony (Bravia e Internet TV), Panasonic (Viera), Samsung (Smart TV) o LG (Google TV) entre otros. Otra opción para acceder a estos servicios son las cajas TDT que se conectan al televisor y le proveen de la señal de TV y la de internet, convirtiéndolo en una televisión conectada.

\section{La televisión conectada es una combina- ción de televisión en el sentido tradicio- nal (broadcast) y banda ancha (broad- band)}

La importancia que este modelo de televisión está cobrando y la falta de estudios publicados sobre la experiencia de uso nos ha llevado a realizar este trabajo con el apoyo y asesoramiento de tres grandes empresas: Lavinia Interactiva, Televisió de Catalunya y Havas Media. En él se analiza la usabilidad y la experiencia de uso de cuatro dispositivos (modelos de 2010) muy distintos entre sí: un televisor que incorpora la conexión a internet, dos cajas TDT con internet que se conectan al televisor, y una videoconsola con servicio de TV a la carta.

Como indicábamos, se trata de un primer estudio para el que no se han encontrado precedentes publicados, lo que lo hace ser pionero en el área. Los trabajos publicados previamente sobre usabilidad en televisión están centrados en televisión interactiva (iTV), un concepto más genérico que no necesariamente implica disponer de internet en el aparato. Como referente en este estudio se ha tenido en cuenta el trabajo sobre diseño de interfaces para TV realizado por Chorianopoulos (2008), así como los que presentan evaluaciones de interfaces de iTV, concretamente los artículos de Pemberton y Griffiths (2003), Chorianopoulos y Spinellis (2006), Obrist, Bernhaupt y Tscheligi (2008), y Pirker y Bernhaupt (2011).

Esta novedad en el objeto de estudio ha llevado a elaborar una metodología propia a partir de los principios de la usabilidad reconocidos por la comunidad de profesionales y académicos de esta área. Esta metodología se compone de dos grandes fases:

a) Análisis de las funciones y análisis heurístico.

b) Tests de usuarios y aplicación de la técnica de seguimiento de la mirada o eye tracking.

Los resultados obtenidos ponen de manifiesto que la usabilidad y la experiencia de usuario son mejorables (al menos tomando como referencia los equipos de 2010), ya que las interfaces confunden a los usuarios y les generan falsas expectativas.

\section{Metodología}

La metodología de investigación principal consiste en los tests de usuarios (user testing). Para preparar las tareas solicitadas a los usuarios se elaboraron dos estudios previos: un análisis de funcionalidades (a través de la técnica del recorrido cognitivo), y una evaluación heurística, para la cual se diseñó una checklist adaptada al objeto de estudio. Los resultados de ambos estudios sirvieron de base para preparar el test de usuarios, y a partir de los resultados de éste se diseñó otra prueba más con tecnología eye tracking haciendo uso del modelo Tobii Glasses.

Se escogieron cuatro equipos siguiendo dos criterios: que estuvieran en el mercado y que fueran muy distintos entre sí. Son los siguientes:

- Televisor Sony Bravia de 42 pulgadas modelo 2010 que incorpora la señal de TDT y la conexión a internet.

- Caja TDT Web:TV de Blu:sens con TDT e internet, un modelo nuevo de fabricación española que como servicio de TV a la carta sólo ofrece canales temáticos, no la programación de las cadenas habituales.

- Caja TDT Engel, que utiliza el estándar HbbTV (Hybrid Broadcast Broadband Television, http://www.hbbtv.org), desarrollado desde 2009 por el consorcio europeo, en el que participan organismos de normalización y de radiodifusión, fabricantes de electrónica, laboratorios, etc. La última regulación de esta norma es la ETSI TS 102796 aprobada en junio de 2010 y modificada en agosto de 2012 en el documento HbbTV Specification Version 1.5.

- Videoconsola PlayStation 3 (PS3), seleccionada por ser distinta a las anteriores; si bien no cuenta con señal TDT, sí dispone de acceso a TV vía internet.

\subsection{Preparación de las pruebas con usuarios: análisis de funcionalidades y evaluación heurística}

El primer análisis tuvo como objetivo conocer de qué funciones disponía cada equipo, y detectar qué dificultades serán las que con mayor probabilidad se encontrarán los usuarios durante las pruebas. En resumen, tres de ellos permiten acceder a la señal TDT (Bravia, Web:TV y Engel), todos ellos disponen de un servicio de TV a la carta (pero en el caso de la Web:TV con contenidos de canales temáticos, no las cadenas más conocidas); dos de ellas disponen de la aplicación de YouTube ya instalada (Bravia y Engel) y también dos tienen un navegador desde el que utilizar el buscador Google (PS3 y Web:TV).

El siguiente paso fue la evaluación heurística. Se trata de un método para analizar la usabilidad de una interfaz (Nielsen; Molich, 1990) en el que, a partir de unos principios de usabilidad aceptados por la comunidad de profesionales y estudiosos del tema, uno o varios expertos en el área revisan el sitio web para detectar posibles problemas de usabilidad. Para llevar a cabo una evaluación de este tipo se utilizan indicadores que concretan los principios heurísticos. Dado que no se encontraron indicadores específicos para el análisis de usabilidad en televisiones conectadas, se creó una checklist ad hoc agrupando los indicadores en hardware, as- 
pectos generales de la interfaz e interfaces de los servicios de TV a la carta. Dentro de cada bloque se incluyeron indicadores relativos a distintos aspectos (puede verse la tabla completa en $h t t p: / / g o o . g l / L V 6 / 4)$

- los indicadores relativos al hardware sirvieron para evaluar el televisor en sí, las cajas de TDT y la consola, así como los respectivos mandos a distancia;

- los relativos a aspectos generales de la interfaz evaluaron los menús, opciones de configuración, teclado del que disponían, secciones de ayuda, y posibilidades en cuanto a la accesibilidad para personas con diversas discapacidades y buscador;

- se crearon indicadores específicos para evaluar los diferentes servicios, como el de la TV con señal TDT, la TV a la carta, la navegación web y otras aplicaciones instaladas por defecto o que puedan instalarse.

La evaluación heurística fue realizada por cuatro personas con conocimientos de usabilidad. Los resultados obtenidos de esta fase sirvieron para preparar los tests de usuarios.

\subsection{Tests de usuarios}

Se seleccionó una muestra de 51 usuarios que comprendieran aproximadamente a partes iguales a hombres y mujeres. 19 eran usuarios jóvenes (18 a 25 años), 20 adultos jóvenes (26 a 35 años), el resto fueron 12 usuarios de entre 35 y 65 años. Prácticamente todos habían oído hablar sobre televisores con conexión a internet, pero no sabían en qué consistían. Ninguno de ellos tenía en casa uno de estos televisores, y sólo 2 personas dijeron que habían usado uno previamente.

Las pruebas se realizaron en una sala de la Universitat Pompeu Fabra preparada a tal efecto y equipada con un sofá, simulando en lo posible un salón de casa.

Debido a que no todos los televisores disponen de las mismas funciones, se optó por estudiar las más representativas y que al menos estuvieran presentes en dos de los modelos estudiados. Las tareas escogidas fueron las siguientes:

- Ver TV en directo: entrar en un canal determinado y localizar en la guía la hora de inicio y fin del programa que se estaba emitiendo (tarea realizada en los equipos Bravia, Web:TV y Engel).

- Ver TV a la carta: los usuarios tenían que localizar el acceso a este servicio, encontrar un programa determinado y un capítulo de éste en concreto, y visionar una parte del programa (tarea realizada en los modelos Bravia, Web:TV, Engel y PlayStation 3).

- Ver un vídeo en YouTube: se trataba de buscar el vídeo más votado de la semana, verlo a pantalla completa y adelantarlo hasta un determinado punto (tarea realizada en Sony Bravia y Engel).

- Hacer una búsqueda determinada en Google (tarea realizada en Web:TV y PlayStation 3).

Cada sesión de las pruebas fue supervisada por un investigador del equipo, mientras que otra persona tomaba notas sobre el tiempo que requería al usuario realizar la tarea, si terminaba o no sin ayuda del moderador, los problemas que provenían del mando a distancia, los que tenían que ver con la interfaz del sistema, y los comentarios que el usuario ha- cía en cada momento. La sesión finalizaba con una entrevista al usuario y un cuestionario.

Los resultados obtenidos en la tarea relativa a la TV a la carta dieron pie a profundizar en este servicio, en particular con el equipo Engel, pues presentaba dificultades que merecía la pena explorar con una prueba específica con tecnología de seguimiento de la mirada.

La usabilidad y la experiencia de usuario son mejorables (al menos con los equipos de 2010), ya que las interfaces confunden a los usuarios y les generan falsas expectativas

\subsection{Test de usuario con eye tracking}

Participaron 20 nuevos usuarios. A todos se les pidió que realizaran la tarea del servicio de TV a la carta con el equipo Engel; tenían que encontrar un programa y localizar un momento determinado.

A diferencia de los otros 3 dispositivos estudiados, el de marca Engel aplica el estándar HbbTV para el acceso a la TV a la carta. Eso significa que no se accede mediante una opción del menú, sino que cuando se está viendo la señal de TDT aparece un aviso en una de las esquinas inferiores de la pantalla indicando que se pulse un botón del mando para acceder a los contenidos de esa cadena en TV a la carta. Con el eye tracker se quiso estudiar si las personas veían el aviso, y en tal caso si lo leían y lo comprendían.

El seguimiento de la mirada en esta prueba se hizo con Tobii Glasses. Se trata de un modelo de eye tracker montado sobre unas gafas; la ventaja es que permite a los usuarios mayor libertad de movimiento que los sistemas que testean desde un monitor. Las grabaciones de los 20 usuarios se analizaron con Tobii Studio 3.0, un software que permite obtener métricas y mapas de las miradas.

En el siguiente apartado se muestran los resultados obtenidos en la evaluación heurística, los tests de usuarios y las sesiones de eye tracking.

\section{Resultados}

\subsection{Evaluación heurística}

A partir de esta evaluación se detectaron los siguientes problemas de usabilidad:

- Los mandos a distancia pueden llevar a confusión por no contar con iconos estándar. En el caso de la PlayStation 3, tener que usarlo con dos manos y contar con un joystick puede ser complicado para algunas personas y tareas.

- El etiquetado de las opciones de los menús de navegación no es intuitivo, es muy probable que alguien clique en una opción esperando que sea algo distinto.

- La ausencia de migas de pan en sistemas con menús tan profundos desorienta durante la navegación.

- Los equipos tienen tiempos de respuesta altos, lo que provoca frustración e impaciencia en los usuarios, acos- 
tumbrados a televisores y ordenadores que responden de forma mucho más rápida a las pulsaciones.

Estos problemas fueron tenidos en cuenta a la hora de planificar las tareas de las pruebas con usuarios.

\subsection{Tests de usuarios}

Para analizar los resultados se tomaron como referencia las tres métricas básicas de la definición de usabilidad de la norma ISO 9241:

- Eficacia: porcentaje de usuarios que realizan cada tarea de forma exitosa sin ayuda del moderador.

- Eficiencia: tiempo promedio que requirieron los usuarios para completar cada tarea. Sólo se contabilizan los usuarios que acaban con éxito.

- Satisfacción: medida con el sistema SUS (system usability scale): se trata de 10 preguntas, 5 positivas y 5 negativas con respecto a la experiencia de uso, que se responden en una escala de Lickert. A cada respuesta se le aplica un cálculo especificado por Brooke (1996). La puntuación mínima que puede obtenerse es 0, la máxima 100.

Se contó con los datos de 51 sesiones repartidas de esta forma: 10 usuarios con Sony Bravia, 18 usuarios con Engel, 11 con Web:TV y 12 con PS3.

\section{Eficacia}

Todos los usuarios, salvo que hubiera problemas técnicos por errores en la señal de TDT o de internet, realizaron todas las tareas previstas para el dispositivo con el que les tocó testear. Cuando el usuario se mostraba perdido y no era capaz de terminar la tarea requerida podía abandonarla y el moderador le ayudaba a encontrar la forma de terminar.

Cuando el usuario finalizaba sin ayuda se consideró que la tarea se había realizado con éxito; si requería ayuda se consideró que no la había finalizado con éxito. El resultado puede verse en la tabla 1: todos los usuarios realizan bien la tarea de búsqueda en YouTube y la mayoría de ellos realizan de forma exitosa la tarea relacionada con TDT, pero las tareas del servicio de TV a la carta y de búsqueda en Google presentan valores más bajos. En el caso de TV a la carta se encuentran los más bajos, entre el $18 \%$ y el $50 \%$.

Las dificultades observadas en la tarea de TV a la carta cambian de un dispositivo a otro. Para empezar, la forma de acceder a este servicio es distinta en los cuatro. En tres de ellos se entra desde el menú principal:

- En el televisor Bravia se accede desde la opción "vídeo", algo que al principio no resultaba evidente porque los usuarios no entendían la TV a la carta como vídeos, aun-

\begin{tabular}{|l|c|c|c|c|}
\hline & TDT & TV a la carta & Google & YouTube \\
\hline Sony Bravia & 100,0 & 50,0 & -- & 100,0 \\
\hline Engel & 87,5 & 35,3 & -- & 100,0 \\
\hline Web:TV & 72,7 & 18,2 & 45,5 & -- \\
\hline PS3 & -- & 50,0 & 75,0 & -- \\
\hline
\end{tabular}

Tabla 1. Eficacia. Porcentaje de usuarios que finalizaron cada tarea con éxito y sin ayuda del moderador que en esencia sí lo son. Una vez localizado, el scroll de cadenas era muy largo y mezclaba las de TV propiamente dichas con otros servicios como cadenas de música y acceso a YouTube.

- En el equipo Web:TV se accede desde la opción "internet". Una vez localizado, al no disponer de canales de TV convencionales costaba identificar que realmente se trataba de un servicio de TV a la carta.

- En la consola PS3 se accede desde la opción "TV". Los usuarios a veces interpretaban que esa opción era para ver televisión por la señal habitual.

El otro dispositivo, el de marca Engel, tenía una forma distinta de acceder a la TV a la carta. Como ya se ha comentado es necesario tener en pantalla la señal de TDT, y a los pocos segundos aparece un aviso en una de las esquinas inferiores de la pantalla indicando que se pulse un botón del mando para acceder a los contenidos de esa cadena en TV a la carta. La mayoría de los usuarios no leían ese aviso porque pensaban que anunciaban algún programa de la cadena. Los que lo leían, lo hacían después de que hubiera aparecido varias veces.

\section{La TV a la carta no ha sido implementada por las TV conectadas de forma que su acceso y uso resulte fácil, directo e intui- tivo}

Las dificultades encontradas en la tarea sobre búsqueda en Google tenían que ver con el teclado que aparecía en pantaIla. Ninguno de los equipos había resuelto bien este aspecto, siendo necesario pulsar las letras una a una usando los cursores del mando para llegar a la deseada.

Un aspecto distinto a los anteriores es el relativo a los mandos a distancia. Los comentarios estaban relacionados con su tamaño: el del televisor Bravia les resultaba excesivamente grande, el de la Web:TV demasiado pequeño; o bien relacionados con los iconos que usaban, en algunos casos poco intuitivos (en especial en Web:TV); y con la disposición de las teclas, que llevaba a pulsar por error (en el televisor Bravia hay dos círculos concéntricos y era frecuente pulsar el que no se quería). Por otro lado, el mando de la consola PS3 generaba expectación al inicio de las tareas, pero por lo general no presentó problemas de uso, a excepción de los usuarios de mayor edad, para quienes resultaba algo muy distinto a lo que habían usado hasta el momento, y que optaron por el sistema de cursores en lugar de hacer uso del joystick que incorpora.

\begin{tabular}{|l|c|c|c|c|}
\hline & TDT & TV a la carta & Google & YouTube \\
\hline Sony Bravia & 1,50 & 3,00 & -- & 1,11 \\
\hline Engel & 1,69 & 4,57 & -- & 1,93 \\
\hline Web:TV & 1,80 & 2,50 & 1,40 & -- \\
\hline PS3 & -- & 4,00 & 1,56 & -- \\
\hline
\end{tabular}

Tabla 2. Eficiencia. Tiempo promedio (en minutos) requerido para cada tarea por los usuarios que terminaron con éxito 


\section{Eficiencia}

La tarea que más tiempo requiere es en todos los casos la que implica acceder al servicio de TV a la carta y buscar un programa en él. Se ha aplicado un t-test para saber si la diferencia de tiempo entre dispositivos es estadísticamente significativa. Con los resultados obtenidos no podemos decir que haya diferencias entre los tiempos obtenidos para las tareas de TDT, TV a la carta ni búsqueda en Google, pero sí se puede afirmar que el tiempo requerido para realizar la tarea de búsqueda en YouTube es mayor en el dispositivo Engel que en el Sony Bravia ( $p$-valor $=0,01)$. La tabla 2 muestra cuánto tiempo tardaron los usuarios en promedio en realizar cada tarea en cada dispositivo.

\section{Satisfacción}

Al finalizar el test se aplicó el cuestionario conocido como SUS. Si seguimos las indicaciones de Jeff Sauro (2011), de los 4 dispositivos sólo uno presenta un nivel aceptable de satisfacción: Sony Bravia. El resto se encuentra por debajo de 68 puntos, límite que establece Sauro para poder afirmar que el producto es mínimamente satisfactorio. De los 4, el equipo Engel es el que menos gustó a los usuarios. La tabla 3 resume las puntuaciones obtenidas.

\subsection{Pruebas con usuarios mediante tecnología de eyetracking}

En este test sólo se analizó la interfaz de la caja TDT Engel. Los 20 usuarios que participaron tuvieron que realizar como tarea acceder a la TV a la carta. Tras 2 minutos para familiarizarse con el mando y con los menús, se les dio un tiempo límite de 5 minutos para realizar la tarea en cada dispositivo.

De las 20 personas que participaron sólo pudieron ser tenidas en cuenta para el análisis 14 de ellas debido a motivos técnicos que hicieron que las grabaciones de algunos usuarios no pudieran recuperarse.

Las métricas evaluadas con el software de eye tracking fueron:

\begin{tabular}{|l|l|}
\hline & SUS \\
\hline Sony Bravia & 75,2 \\
\hline Engel & 50,0 \\
\hline Web:TV & 62,7 \\
\hline PS3 & 66,5 \\
\hline
\end{tabular}

Tabla 3. Satisfacción. Puntuación del cuestionario SUS

- Número de veces que aparece el aviso en pantalla para acceder a la televisión a la carta: apareció como mínimo una vez y como máximo 7 por cada participante, es decir, que casi todos los usuarios tuvieron varias oportunidades para verlo.

- Tiempo de exposición en pantalla del aviso: entre 5 y 21 segundos, dependiendo de cuántas veces apareciera y el tiempo que el usuario tardara en cambiar a otra pantalla.

- Número de usuarios que fijaron la mirada en el aviso: sólo 4 de los 14 participantes lo hicieron (el eye traker lo detecta a partir de la visión foveal, que es la visión más nítida de las personas).

- Tiempo que tardan estos 4 usuarios desde que aparece el aviso hasta que lo ven: en promedio fue de 1,64 segundos.

- Número de fijaciones que estos 4 usuarios realizan sobre el aviso: varía entre 1 y 11, según cada usuario.

- Duración de la fijación ocular de los 4 usuarios en el aviso: el que más tiempo dedica a ver el aviso está en 2,21 segundos, mientras que el que menos le dedica sólo 0,27 segundos.

- Número de personas que acceden finalmente a la TV a la carta: sólo 1 usuario de los 4 que vieron el aviso reconoció que se trataba de las indicaciones para el acceso al servicio.

Los resultados obtenidos con el análisis del comportamiento ocular muestran claramente que la fórmula del aviso en pantalla es poco clara, que los usuarios difícilmente lo ven y que incluso viéndolo no saben interpretar cuál es la

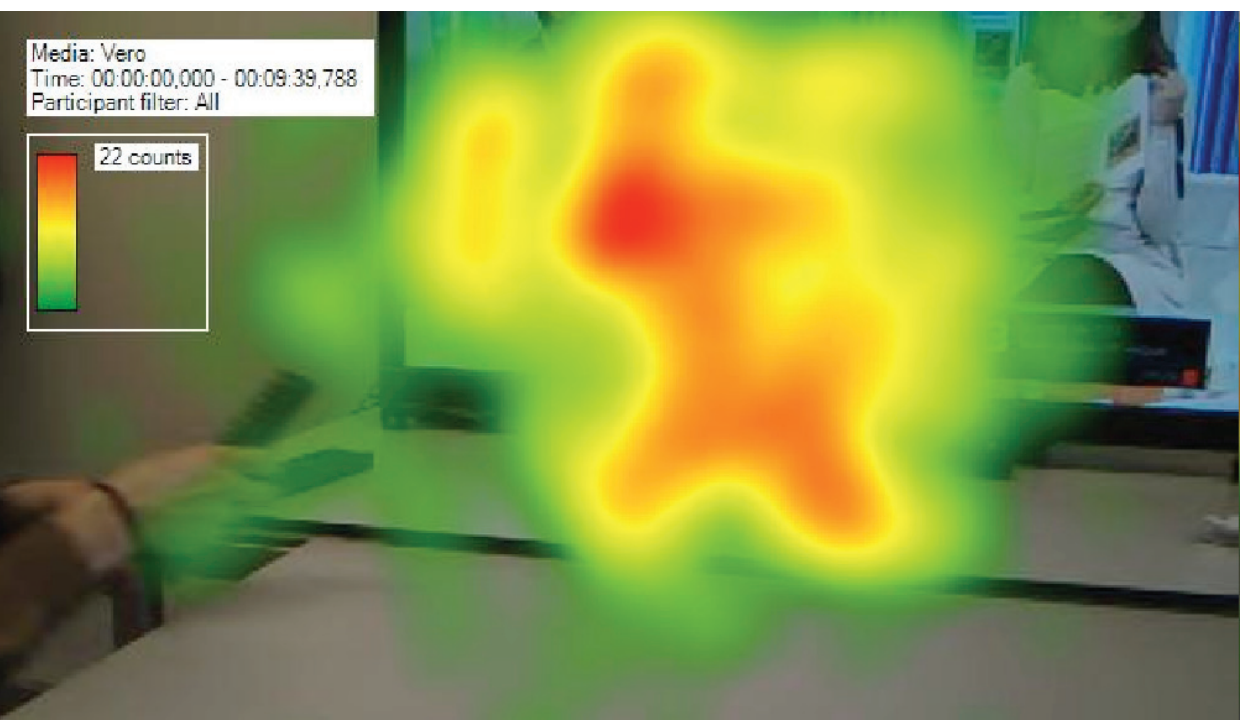

Figura 1. Mapa de calor generado por Tobii Studio de los 14 usuarios sumando todos los momentos en los que aparece el aviso en la esquina inferior derecha. El color rojo indica las áreas donde más miradas ha habido, y se va degradando hacia amarillo y verde según disminuye el número de miradas. forma de acceder al servicio de TV a la carta. La figura 1 muestra el mapa de calor de los 14 usuarios en el momento en que el aviso está en pantalla. Puede observarse cómo apenas dirigen la mirada a él (en rojo se marcan las zonas más vistas).

\section{Conclusiones}

A partir de los resultados obtenidos a lo largo de las distintas metodologías empleadas en este estudio, y con las conversaciones mantenidas con los usuarios una vez finalizadas las pruebas, queda claro que uno de los servicios que genera más ex- 
pectativas, la TV a la carta, no ha sido implementado por las TV conectadas de forma que su acceso y uso resulte fácil, directo e intuitivo.

Los comentarios más generalizados de los usuarios eran de frustración. Acostumbrados a la inmediatez de los contenidos de la TV y la multitarea del ordenador, les parecía extraño que los televisores conectados no sumaran las ventajas ambos.

Los usuarios manifestaron que por ahora no comprarían un televisor conectado porque las funciones que ofrecen son similares a las que consiguen viendo su televisor tradicional mientras usan el portátil conectado a internet.

Los equipos presentaron deficiencias que generaron confusión y la consiguiente frustración. Falta de iconografía estándar en el mando a distancia, menús poco intuitivos, dificultad en el uso del teclado, confusión en el acceso a VoD y dificultad para navegar por internet fueron los aspectos más reseñables obtenidos en estas evaluaciones.

Hay que tener en cuenta que en este estudio se analizaron sólo 4 dispositivos de los muchos que hay en el mercado, y que las pruebas se hicieron sobre modelos de 2010, por lo que los resultados obtenidos hay que contextualizarlos y no necesariamente son generalizables a todas las televisiones conectadas ni a las más modernas. De hecho, algunos fabricantes han incorporado elementos que no están presentes en este estudio, como teclados en el mando o en la pantalla que facilitan el ingreso de palabras en el sistema, o como la integración del móvil con la televisión mediante aplicaciones específicas.

Estas limitaciones no quitan validez al estudio, ya que la metodología aplicada es rigurosa y ha servido para constatar que los errores de usabilidad pueden frenar el uso de nuevos dispositivos, en este caso tan populares como la televisión.

Los errores de usabilidad pueden frenar el uso de nuevos dispositivos

\section{Agradecimientos}

Esta investigación no hubiera sido posible sin Javier Díaz, David Hernández y Jaume Ponsa, que ayudaron en las sesiones de tests de usuarios y en la evaluación heurística. Y por supuesto, nuestro agradecimiento a los usuarios que participaron en el estudio.

\section{Bibliografía}

Brooke, John (2005). "SUS: A "quick and dirty" usability scale". In Jordan, P. W.; Thomas, B.; Weerdmeester, B. A.; McClelland, A. L. Usability evaluation in industry. London: Taylor and Francis.

http://www.itu.dk/courses/U/E2005/litteratur/sus.pdf

Chorianopoulos, Konstantinos (2008). "User interface design principles for interactive television applications". International journal of human-computer interaction, July, v. 24, n. 6. pp. 556-573.

http://dx.doi.org/10.1080/10447310802205750

Chorianopoulos, Konstantinos; Spinellis, Diomidis (2006). "User interface evaluation of interactive TV: a media studies perspective". Universal access in the information society, July, v. 5, n. 2, pp. 209-218.

http://dx.doi.org/10.1007/s10209-006-0032-1

El mundo (2007). “Gates augura que la red revolucionará la televisión en los próximos cinco años". El mundo, 30 enero. http://www.elmundo.es/navegante/2007/01/29/ tecnologia/1170063702.html

HbbTV (2012). "HbbTV specification version 1.5". http://www.hbbtv.org/pages/about_hbbtv/HbbTVspecification-1-5_Aug2012.pdf

Nielsen, Jakob; Molich, Rodolf (1990). “Heuristic evaluation of user interfaces". En: ACM CHI, April, pp. 249-256. http://dx.doi.org/10.1145/97243.97281

Pirker, Michel; Bernhaupt, Regina (2011). “Measuring user experience in the living room: results from an ethnographically oriented field study indicating major evaluation factors". En: 9th intl interactive conf on interactive television, pp. 79-82.

http://dx.doi.org/10.1145/2000119.2000133

Obrist, Marianna; Bernhaupt, Regina; Tscheligi, Manfred (2008). "Interactive TV for the home: an ethnographic study on users' requirements and experiences". Intl journal of human-computer interaction, Feb., v. 24, n. 2, pp. 174-196. http://dx.doi.org/10.1080/10447310701821541

Pemberton, Lyn; Griffiths, Richard (2003). “Usability evaluation techniques for interactive television". En Stephanidis, C. (ed.). Procs of human-computer interaction intl, Universal access in $\mathrm{HCl}$ : inclusive design in the information society. Lawrence Erlbaum Associates, v. 4, pp. 882-886.

Sauro, Jeff (2011). "Measuring usability with the system usability scale (SUS)". Measuring usability, 2 Febr. http://www.measuringusability.com/sus.php 